\title{
Anestesia peribulbar com ropivacaína como alternativa ao bloqueio neuromuscular para facectomia em cães
}

\author{
[Peribulbar anesthesia with ropivacaine as an alternative to neuromuscular blocking agents for \\ cataract surgery in dogs] \\ V.N.L.S. Oliva ${ }^{1}$, A.L. Andrade ${ }^{1}$, L. Bevilacqua ${ }^{2}$, L.M. Matsubara ${ }^{2}$, S.H.V. Perri ${ }^{1}$ \\ ${ }^{1}$ Curso de Medicina Veterinária - UNESP/Araçatuba \\ Rua Clóvis Pestana, 793 \\ 16050-680 - Araçatuba, SP \\ ${ }^{2}$ Aluna de pós-graduação - FM-UNESP - Botucatu, SP
}

\begin{abstract}
RESUMO
Desenvolveu-se uma técnica de bloqueio peribulbar comparando-a à técnica de anestesia oftálmica com bloqueio neuromuscular parcial em cães submetidos à facectomia extracapsular. Doze cães, de diferentes raças, foram alocados em dois grupos (G1 e G2) e anestesiados com acepromazina $(0,05 \mathrm{mg} / \mathrm{kg}$, IV) e propofol $(5 \mathrm{mg} / \mathrm{kg}$, IV) e mantidos com isofluorano sob ventilação espontânea. Os cães do G1 receberam o bloqueio peribulbar com ropivacaína $0,75 \%$, e os do G2 o bloqueio neuromuscular parcial com brometo de pancurônio $(0,01 \mathrm{mg} / \mathrm{kg}$, IV). Utilizaram-se, como parâmetros comparativos, escores de posicionamento do globo ocular, controle do reflexo oculocardíaco e pressão intraocular $(\mathrm{mmHg})$. Foi possível propor uma técnica eficaz para cães baseando-se na técnica de bloqueio peribulbar posterior realizada no homem. Em ambos os grupos, o globo ocular permaneceu centralizado. Não ocorreram alterações eletrocardiográficas atribuídas ao reflexo oculocardíaco. Houve redução significativa da

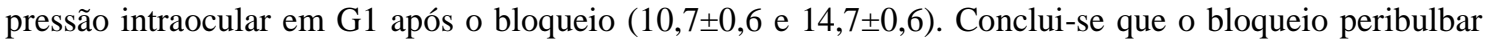
proporcionou condições cirúrgicas apropriadas para a realização da facectomia, com a vantagem de promover bloqueio sensitivo do olho.
\end{abstract}

Palavras-chave: cão, bloqueio peribulbar, ropivacaína, brometo de pancurônio, facectomia

\begin{abstract}
A peribulbar block technique was developed and its clinical efficacy was compared with neuromuscular blockade in dogs undergoing cataract surgery. Twelve dogs of different breeds were randomly and equally allocated in two groups. After given acepromazine $(0.05 \mathrm{mg} / \mathrm{kg}, \mathrm{IV})$, anesthesia was induced with propofol $(5 \mathrm{mg} / \mathrm{kg}, \mathrm{IV})$ and maintained with isoflurane in oxygen during spontaneous breathing. A peribulbar block with $0.75 \%$ ropivacaine was performed in G1 dogs while partial neuromuscular blockade with pancuronium $(0.01 \mathrm{mg} / \mathrm{kg} I V)$ was provided in G2 dogs. Globe position scores, oculocardiac reflex, and intra-ocular pressure $(\mathrm{mmHg})$ were evaluated at pre-defined intervals during surgery. Peribulbar blocks were successful performed according to posterior peribulbar block described in humans. In both groups, the globe was centralized and globe position scores did not differ between groups. The intra-ocular pressure was significantly lower in G1 after the block $(10.7 \pm 0.6$ vs $14.7 \pm 0.6)$. There were no electrocardiographycal changes attributed to the oculocardiac reflex. In conclusion, satisfactory surgical conditions were provided by the peribulbar block. This technique can be used as an alternative to the use of neuromuscular blocking agents in dogs undergoing cataract surgery, with the advantage of providing analgesia of the eye.
\end{abstract}

Keywords: dog, peribulbar block, ropivacaine, pancuronium bromide, cataract surgery

Recebido em 2 de julho de 2009

Aceito em 2 de junho de 2010

E-mail: voliva@fmva.unesp.br 


\section{INTRODUÇÃO}

Vários fatores devem ser considerados em um procedimento anestésico para cirurgia intraocular, particularmente na facectomia, pois podem ser diretamente afetados pelo agente ou pela técnica anestésica utilizada, interferindo na resposta terapêutica. Nesses pacientes, é necessário manter a pressão intraocular (PIO) em valores próximos do normal, promover dilatação pupilar, prevenir a ativação do reflexo oculocardíaco (ROC), imobilizar a musculatura extraocular a fim de determinar um campo cirúrgico adequado e, por fim, propiciar recuperação anestésico-cirúrgica suave e com suporte analgésico adequado (Thurmon et al., 1996). Em animais de pequeno porte, é possível estabelecer condições cirúrgicas apropriadas com diferentes associações de fármacos para a indução e a manutenção da anestesia.

Durante a manutenção anestésica, recomenda-se a utilização dos bloqueadores neuromusculares (BNM) não despolarizantes, para promover relaxamento da musculatura extraocular e centralização do globo, evitando-se planos anestésicos profundos com depressão cardiorrespiratória muito acentuada (Ludders, 2003; Carregaro et al., 2006). Além disso, a paralisia resulta no posicionamento centralizado do globo ocular, diminuindo a manipulação cirúrgica sobre esse e eliminando a chance de ocorrência do reflexo oculocardíaco (Clutton et al., 1988).

Estes fármacos podem ser utilizados em doses tituladas para promover relaxamento da musculatura extraocular em cirurgias intraoculares, sem causar depressão ventilatória do paciente (Carregaro et al., 2006). Lee et al. (1998) obtiveram paralisia muscular seletiva com brometo de pancurônio na dose de $0,01 \mathrm{mg} / \mathrm{kg} \mathrm{IV}$, entretanto ressaltaram a importância do monitoramento intensivo e do suporte ventilatório quando necessário.

Na medicina humana, a utilização da anestesia regional orbitária para cirurgia oftálmica tornou-se uma prática rotineira nos últimos anos (Vanetti, 2001). A anestesia peribulbar foi primeiramente relatada no homem por Davis e Mandel, em 1986. Esse bloqueio é também denominado de "bloqueio extraconal", uma vez que a agulha é introduzida fora do cone muscular extraocular (Troll, 1995). A técnica tradicional e mais comumente utilizada restringe-se basicamente à de dupla punção, entretanto várias modificações foram realizadas no protocolo original (McGoldrick et al., 2001). Uma variação eficiente dessa técnica é a peribulbar posterior, na qual a solução anestésica é depositada com uma agulha mais longa posteriormente ao globo ocular, fora do cone muscular (Vanetti, 2001).

Os bloqueios orbitários promovem efeitos benéficos mesmo quando associados à anestesia geral. Em trabalho realizado por Déb et al. (2001), a associação do bloqueio peribulbar à anestesia geral inalatória em cirurgias oculares em crianças promoveu analgesia intra e pós-operatória adequadas, evidenciadas pela estabilidade hemodinâmica transoperatória e pela recuperação pós-anestésica suave. Adicionalmente, o bloqueio reduziu a incidência do reflexo oculocardíaco e a necessidade de suplementação de analgésicos opioides no período de recuperação.

Diversos anestésicos locais podem ser utilizados na realização do bloqueio peribulbar no homem; entre eles, a ropivacaína, em diferentes concentrações, tem demonstrando melhor perfil para cirurgia oftálmica. Esse fármaco parece oferecer vantagens por influenciar o volume sanguíneo intraocular e, por consequência, a redução da PIO, devido ao seu efeito vasoconstritor próprio (Nociti et al., 2001; Olmez et al., 2004). Sua eficácia clínica foi comprovada por diferentes autores quando utilizada na concentração de $0,75 \%$ (Vásquez et al., 2002; Gioia et al., 2004).

Há poucos relatos sobre a utilização dos bloqueios regionais orbitários em cães. Kanemaki et al. (2000) e Accola et al. (2006) relataram a utilização da anestesia retrobulbar como uma alternativa à utilização dos BNM em cirurgias de córnea e intraoculares para promover acinesia ocular e, adicionalmente, ressaltam a vantagem dessa técnica em promover analgesia local residual.

O objetivo deste trabalho foi desenvolver uma técnica de bloqueio regional peribulbar e avaliar sua eficácia durante a facectomia extracapsular em cães, bem como comparar o bloqueio regional peribulbar com a técnica anestésica tradicional.

\section{MATERIAL E MÉTODOS}

Doze cães com catarata madura bilateral, de diferentes raças e sexos (quatro machos e oito fêmeas), com média de idade de 6,7 $\pm 3,0$ anos e média de peso de $8,4 \pm 4,7 \mathrm{~kg}$, foram submetidos à facectomia extracapsular realizada pelo mesmo cirurgião. Todos os animais foram submetidos à avaliação pré-operatória, sendo incluídos no estudo somente aqueles que se enquadraram nas exigências estipuladas para realização da 
facectomia extracapsular, seguindo os critérios propostos por Gilger (2003).

Os cães foram distribuídos aleatoriamente em dois grupos experimentais G1 e G2. A medicação pré-anestésica foi realizada com a acepromazina a $0,2 \%(0,05 \mathrm{mg} / \mathrm{kg}$ IV) (Univet S.A., São Paulo, Brasil) e, decorridos 15 minutos, foi realizada a punção da veia cefálica com cateter (BD Angiocath ${ }^{\mathrm{TM}}$ 22GAx1,00 IN Becton, Dickinson Ind. Cir. Ltda., Juiz de Fora, Brasil) para fluidoterapia transoperatória com solução de Ringer com lactato de sódio $(10 \mathrm{~mL} / \mathrm{kg} / \mathrm{h})$ seguida da indução anestésica com propofol (Cristália Prod. Quím. Farm. Ltda., Itapira, Brasil) $(5 \mathrm{mg} / \mathrm{kg}$ IV). Após a intubação traqueal (Tubo endotraqueal com manguito Solidor $^{\circledR}$ - Medico Int. Trading Co. Ltda., Tianjin P.R., China) em decúbito lateral, foi realizada conexão a um circuito anestésico (Aparelho de anestesia Origami Ergo - K. Takaoka Ind. e Com. Ltda., São Paulo, Brasil) adequado ao porte do animal. Os animais com peso inferior a $7 \mathrm{~kg}$ foram conectados ao circuito de anestesia em sistema sem reinalação, utilizando-se um fluxo diluente de $\mathrm{O}_{2}$ a $100 \%$ de $200 \mathrm{~mL} / \mathrm{kg} / \mathrm{min}$. Os animais acima desse peso foram conectados a um circuito circular valvular em sistema com reinalação parcial, com fluxo diluente de $\mathrm{O}_{2}$ a $100 \%$ de $50 \mathrm{~mL} / \mathrm{kg} / \mathrm{min}$. Todos os animais foram mantidos em ventilação espontânea, com possibilidade de instituir ventilação controlada (Ventilador Eletrônico 668 (Sevo) - K. Takaoka Ind. e Com. Ltda., São Paulo, Brasil) nos casos de apneia ou quando a pressão parcial de dióxido de carbono no sangue arterial (PaCO2) (Roche OMNIC - Roche Diagnostics - Mannheim, Alemanha) atingisse valor maior que $50 \mathrm{mmHg}$.

A administração do agente anestésico isofluorano (Cristália Prod. Quím. Farm. Ltda.) foi obtida utilizando-se um vaporizador universal microprocessado (Multiagente 1415 Plus - K. Takaoka Ind. e Com. Ltda.). Foi estipulado que a concentração do anestésico seria aumentada se a pressão arterial média se elevasse $30 \%$ do valor prévio obtido durante a cirurgia, ou diminuída se a pressão arterial média se reduzisse abaixo de $70 \mathrm{mmHg}$.

Decorridos 20 minutos do início da vaporização do agente anestésico inalatório, foi realizado o bloqueio peribulbar com ropivacaína (Astra
Zeneca do Brasil Ltda., Cotia, Brasil) a 0,75\% nos cães do G1 e o bloqueio neuromuscular parcial com brometo de pancurônio (Cristália Prod. Quím. Farm. Ltda.) (0,01mg/kg IV) nos cães do G2. A cirurgia teve início após 20 minutos da realização do bloqueio peribulbar em G1 e cinco minutos após a administração do bloqueador neuromuscular em G2.

Realizou-se a técnica de bloqueio peribulbar posterior com dupla punção transconjuntival descrita para o homem, de acordo com McGoldrick et al. (2001). Utilizou-se agulha hipodérmica descartável $0,7 \mathrm{~mm} \times 25 \mathrm{~mm}$ (BD Precision Guide ${ }^{\circledR}$ - Becton Dickinson Ind. Cir. Ltda.) (22Gx1"), a qual foi previamente marcada com uma caneta com a medida do comprimento do globo ocular do paciente, obtido previamente por ultrassonografia (Portable Linear/ConvexScanning Ultrasonic Diagnostic Sistem - Model CTS-385V - SIUI - Santon Institute of Ultrassonic Instruments - Convex Probe 7,5MHz), para determinar sua profundidade de inserção (espaço peribulbar posterior - posteriormente ao globo ocular). A agulha foi inserida primeiramente através da conjuntiva em um ponto situado no terço lateral na margem orbitária inferior e em um segundo ponto situado no terço medial da margem orbitária superior. Ambas em sentido anteroposterior, na tentativa de abordar o espaço peribulbar posterior injetando-se o anestésico lentamente durante um minuto fora do cone muscular (Fig.1 e 2). Teve-se o cuidado de manter a agulha mais próxima à parede orbitária durante sua inserção, para evitar perfuração ocular e manter o bisel direcionado para o globo ocular, a fim de reduzir resistência durante a administração do anestésico local. O volume de anestésico local administrado em cada ponto de punção variou conforme a tolerância e a distensibilidade do espaço orbitário, como citado por Vásquez et al. (2002), com o cuidado de se evitar a ocorrência de exoftalmia e o aumento excessivo da pressão intraocular. Após cada injeção, realizou-se compressão digital da órbita durante um minuto, para auxiliar na difusão da solução anestésica.

No período transanestésico, foram avaliadas: as frequências cardíaca (FC) e respiratória (FR), mensuradas com estetoscópio esofágico; a concentração anestésica média (CA), utilizandose um vaporizador universal microprocessado 
(Multiagente 1415 Plus - K. Takaoka Ind. e Com. Ltda.) a pressão arterial média invasiva (PAM), mensurada por método invasivo inserindo um cateter (BD Angiocath ${ }^{\mathrm{TM}}$ 22GAx1,00 IN Becton, Dickinson Ind. Cir. Ltda.) na artéria femoral e conectando-o a um manômetro (Heidjii - São Paulo, Brasil) aneroide; a saturação de oxigênio na hemoglobina $\left(\mathrm{SpO}_{2}\right)$, mensurada com oxímetro de pulso (Oxilife Plus - CMOS Drake, Ilhéus, Brasil); o ritmo cardíaco, avaliado pelos registros eletrocardiográficos (TEB C10 Tec. Elet. Bras. Ltda., São Paulo, Brasil) (ECG) realizados na derivação II; e a hemogasometria (Roche OMNIC - Roche Diagnostics GMBH), realizada de amostras de sangue arterial colhidas anaerobicamente em seringa heparinizada da artéria femoral. Todos os dados citados foram obtidos a cada cinco minutos após o início da vaporização anestésica, exceto os registros eletrocardiográficos e as análises hemogasométricas, que foram realizados em intervalos predefinidos durante a cirurgia, juntamente com os parâmetros comparativos.

Para análise comparativa dos grupos, avaliaramse os escores de rotação do globo ocular determinados em relação ao eixo neutro de visão, utilizando-se um goniômetro conforme Lee et al. (1998). A pressão intraocular foi mensurada com tonômetro de Schiotz (Schiotz Tonometer Inclined Sacle Improved Model) utilizando peso de 5,5g e empregando-se uma tabela de conversão para cães, segundo Morgan (1997). A deflagração do reflexo oculocardíaco (ROC) foi monitorada por análise dos registros eletrocardiográficos.

Estes dados foram obtidos nos seguintes momentos (M) em G1: M0 - antes da medicação pré-anestésica; M1 - 15 minutos após M0: indução anestésica, seguida da intubação traqueal e da manutenção anestésica; M2 - 20 minutos após M1, realização do bloqueio peribulbar (G1) ou aplicação do BNM (G2); M3 - 20 minutos após M2 (G1 - período de latência do bloqueio peribulbar), cinco minutos após M2 (G2 período de latência do BNM), M4 - incisão corneal; M5 - remoção do cristalino; M6 - início da sutura corneal; e M7- término da cirurgia.

Também foram avaliados: o volume total de ropivacaína administrado no espaço peribulbar volume total administrado nos dois pontos de punção -, o período de latência do bloqueio motor - início da centralização do globo ocular -
, o período de ação do bloqueio motor momento de obtenção de acinesia do globo ocular até o retorno de sua movimentação - e o período de ação do bloqueio sensitivo, iniciado 15 minutos após a realização do bloqueio peribulbar - verificação de reflexo corneal, testado por um toque sobre a córnea com suabe estéril.

O fármaco analgésico utilizado no período pósoperatório foi o cloridrato de tramadol (Searle Monsanto do Brasil Ltda., São Paulo, Brasil), $2 \mathrm{mg} / \mathrm{kg}$ IM, administrado ao término do bloqueio sensitivo promovido pela anestesia peribulbar em G1 e cinco minutos antes do final da cirurgia em G2. A analgesia complementar e o controle da inflamação ocular foram realizados com carprofeno (Laboratórios Pfizer Ltda., Guarulhos, Brasil), 4mg/kg VO, a cada 24 horas.

Os dados referentes à PIO foram submetidos à análise de variância com medidas repetidas, sendo as médias dos grupos comparadas por meio do teste Tukey, e os momentos pela análise de regressão, sendo testados quanto à normalidade e à homogeneidade de variâncias. Os dados de escores do grau de rotação do globo ocular foram analisados usando-se a análise de variância não paramétrica de Friedman para comparar momentos em cada grupo e o teste de Mann-Whitney para comparar grupos em cada momento. As análises estatísticas foram efetuadas empregando-se o programa SAS/1999 com índice de significância de $\mathrm{P}<0,05$.

\section{RESULTADOS E DISCUSSÃO}

O tempo médio de duração das cirurgias foi de $37 \pm 4,1$ e $40 \pm 6,8$ minutos, e o tempo anestésico total médio foi de $115 \pm 6,3$ e $78 \pm 5,2$ minutos no G1 e G2, respectivamente. O tempo anestésico mais prolongado observado em G1 pode ser atribuído ao período de latência do bloqueio peribulbar.

Os valores dos parâmetros cardiorrespiratórios (Tab. 1) demonstram que o procedimento anestésico em ambos os grupos foi adequado e manteve níveis esperados para a espécie. Os valores de frequência respiratória, da $\mathrm{PaCO}_{2}$ e do $\mathrm{pH}$ arterial não evidenciaram acidose respiratória, ao contrário dos resultados apresentados por Lee et al. (1998), que utilizaram a mesma dose de brometo de pancurônio. 
Tabela 1. Parâmetros de monitoração anestésica (valores máximo e mínimo) em cães anestesiados pelo isofluorano em associação ao bloqueio peribulbar com ropivacaína a $0,75 \%$ (G1) e ao bloqueio neuromuscular com brometo de pancurônio $(0,01 \mathrm{mg} / \mathrm{kg}$ IV) $(\mathrm{G} 2)$

\begin{tabular}{lcccc}
\hline Parâmetro & \multicolumn{2}{c}{$\mathrm{G} 1(\overline{\mathrm{X}} \pm \mathrm{EPM})$} & \multicolumn{2}{c}{$\mathrm{G} 2(\overline{\mathrm{X}} \pm \mathrm{EPM})$} \\
\hline $\mathrm{FC}(\mathrm{bpm})$ & $100 \pm 5$ & $119 \pm 6$ & $113 \pm 4$ & $146 \pm 5$ \\
$\mathrm{FR}(\mathrm{mpm})$ & $13 \pm 1$ & $45 \pm 2$ & $15 \pm 1$ & $50 \pm 5$ \\
$\mathrm{PAM}(\mathrm{mmHg})$ & $82 \pm 1$ & $90 \pm 1$ & $66 \pm 2$ & $77 \pm 2$ \\
$\mathrm{CA}(\mathrm{V} \%)$ & $1,67 \pm 0,1$ & $2,3 \pm 0,1$ & $2,7 \pm 0,1$ & $3,0 \pm 0,1$ \\
$\mathrm{SpO}_{2}(\%)$ & $96,8 \pm 0,1$ & $98,0 \pm 0,2$ & $97,2 \pm 0,3$ & $97,5 \pm 0,1$ \\
$\mathrm{Ph}$ & $7,35 \pm 0,01$ & $7,39 \pm 0,01$ & $7,35 \pm 0,01$ & $7,41 \pm 0,01$ \\
$\mathrm{PaCO}_{2}(\mathrm{mmHg})$ & $31,3 \pm 0,2$ & $36,6 \pm 1,0$ & $32,6 \pm 0,7$ & $40,7 \pm 1,0$ \\
$\mathrm{HCO}_{3}(\mathrm{mmol} / \mathrm{L})$ & $18,7 \pm 0,1$ & $19,6 \pm 0,2$ & $20,0 \pm 0,5$ & $21,6 \pm 0,5$ \\
$\mathrm{PaO}_{2}(\mathrm{mmHg})$ & $100,1 \pm 1$ & $481,1 \pm 12$ & $98 \pm 2$ & $436,7 \pm 10$ \\
$\mathrm{SO}_{2}(\%)$ & $97,0 \pm 0,01$ & $99,9 \pm 0,01$ & $96,7 \pm 0,16$ & $99,9 \pm 0,01$ \\
$\mathrm{BE}^{(m m o l . / L)}$ & $4,6 \pm 0,2$ & $4,7 \pm 0,5$ & $3,1 \pm 0,4$ & $4,2 \pm 0,2$ \\
\hline
\end{tabular}

FC: frequência cardíaca; FR: frequência respiratória; PAM: pressão arterial média; CA: concentração anestésica média do isofluorano no vaporizador; $\mathrm{PaCO}_{2}$ : saturação de oxigênio na hemoglobina; $\mathrm{pH}$ : potencial hidrogeniônico; $\mathrm{PaCO}_{2}$ : pressão parcial de dióxido de carbono no sangue arterial; $\mathrm{HCO}_{3}$ : concentração de bicarbonato no sangue arterial; $\mathrm{PaO}_{2}$ : pressão parcial de oxigênio no sangue arterial: $\mathrm{SO}_{2}$ : saturação de oxigênio na hemoglobina no sangue arterial; BE: déficit de base no sangue arterial.

Foi possível desenvolver uma técnica de anestesia peribulbar para cães fundamentando-se na técnica de bloqueio peribulbar posterior realizada no homem, constatada como eficiente por Vásquez et al. (2002). Os bloqueios foram realizados com sucesso utilizando-se agulha hipodérmica descartável $0,7 \mathrm{~mm} \times 25 \mathrm{~mm}$, assim como descrito em diferentes trabalhos realizados na anestesia humana (Perello et al., 2000; Déb et al., 2001; Olmez et al., 2004).

O volume total de ropivacaína administrado em cada bloqueio variou de 2 a $4,3 \mathrm{~mL}$. Para a realização do bloqueio peribulbar no homem, são necessários grandes volumes de solução anestésica ( 8 a $12 \mathrm{~mL})$, pois seu efeito depende da dispersão do anestésico local pela órbita para atingir o interior do cone muscular extraocular e anestesiar os nervos aí contidos (Troll, 1995). Contudo, frente aos resultados observados em um ensaio prévio realizado em cães, verificou-se que aqueles volumes foram excessivos quando utilizados na região peribulbar do cão, causando acentuada exoftalmia e aumento da pressão intraocular. Além disso, os volumes injetados em cada ponto de punção variaram em cada paciente frente às diferenças do tamanho orbitário nas várias raças selecionadas para o estudo. Assim, neste trabalho, procurou-se administrar volumes de acordo com a tolerância desse espaço, como citado por Vásquez et al. (2002) e Olmez et al. (2004), evitando-se a ocorrência de exoftalmia e o aumento excessivo da pressão intraocular. Isto foi possível por meio da avaliação da tensão e do grau de mobilidade do globo ocular e da pálpebra superior, mediante a palpação ocular durante a administração do anestésico local.

O volume de ropivacaína administrado ultrapassou a dose terapêutica de $3 \mathrm{mg} / \mathrm{kg}$ (Skarda, 1996) na maioria dos pacientes do G1. Além disso, ultrapassou-se a dose tóxica convulsivante de 4,9mg/kg (Skarda, 1996) em um dos animais desse grupo. Entretanto, não houve indícios de neurotoxicidade ou cardiotoxicidade durante os períodos cirúrgico e pós-operatório, provavelmente devido à ação vasoconstritora intrínseca desse anestésico, como descrito no trabalho de Ishiyama et al. (1997), característica que poderia diminuir ou tornar mais lenta sua absorção pelos vasos sanguíneos orbitários. Dessa forma, poder-se-ia sugerir que, nos bloqueios peribulbares, em cães, a ropivacaína fosse utilizada em concentrações menores, o que permitiria o uso de volumes maiores e resultados satisfatórios, como observado por Perello et al. (2000).

No presente estudo, decidiu-se pela não realização da compressão do globo ocular após a realização do bloqueio peribulbar, com base nos relatos de Ling et al. (2001), que demonstraram que a ausência desta não resulta em valores elevados de PIO. Assim, optou-se por realizar uma leve compressão digital de apenas um minuto após a aplicação do anestésico local em 
cada ponto de punção do bloqueio, a fim de facilitar a difusão do anestésico, o que se mostrou eficaz.

O período estipulado para o início da cirurgia, 20 minutos após a realização do bloqueio, foi determinado baseando-se no período de latência para os bloqueios motor e sensitivo completos promovidos pela ropivacaína a $0,75 \%$ quando utilizada em bloqueio peribulbar no homem de 10 a 15 minutos, segundo Vásquez et al. (2002) e Gioia et al. (2004).

O período de latência de bloqueio motor proporcionado pelo bloqueio peribulbar foi de um minuto após a administração da ropivacaína no primeiro ponto de punção em todos os pacientes estudados. Esse período foi menor que os observados em trabalhos que testaram a ropivacaína a $0,75 \%$ na mesma técnica, no homem (Olmez et al., 2004). O bloqueio precoce observado neste trabalho pode ser consequente à presença de relaxamento prévio da musculatura extraocular promovida pela anestesia geral, adicionada à característica do isoflurano em promover qualidade de relaxamento muscular e centralização ocular superiores aos promovidos pelo halotano, como citado por Hodgson e Dunlop (1990).

O período médio de ação do bloqueio motor e sensitivo promovido pelo bloqueio peribulbar foi de $133 \pm 56$ minutos e $272 \pm 64$ minutos, respectivamente. Tal diferença deve-se, provavelmente, ao fato de a ropivacaína ter maior afinidade por fibras sensitivas $\mathrm{A} \delta$ e $\mathrm{C}$ do que por fibras motoras, como demonstrado nos trabalhos de Feldman e Covino (1988). Esse bloqueio sensitivo mais duradouro foi positivo, pois determinou analgesia local residual com recuperação pós-anestésica suave.

Os dois protocolos propostos propiciaram posicionamento do globo ocular adequado para a cirurgia, não havendo diferença significativa entre os grupos, como demonstrado na Tab. 2.

A maioria dos pacientes do G1 apresentou posicionamento ocular desejável desde o início da cirurgia até a remoção do cristalino (M4 e M5) (Fig. 3). Entretanto, um paciente (17\%) apresentou leve desvio ocular inferior ao final da realização do bloqueio peribulbar, e, ao final do procedimento cirúrgico, momentos referentes à sutura corneal (M6 e M7), houve posicionamento ocular indesejável.

A resposta insatisfatória apresentada por esse paciente poderia ter sido revertida com dose suplementar de anestésico local depois de concluído o período de latência do bloqueio. Troll (1995) afirma que anestesia peribulbar normalmente necessita de injeções suplementares do anestésico local, uma vez que seu efeito depende da difusão da solução anestésica através do septo intermuscular para atingir o interior do cone muscular.

Tabela 2. Valores da mediana (Md), mínimo (Min) e máximo (Max) dos escores relacionados ao grau de rotação do globo ocular, em cães anestesiados pelo isofluorano em associação ao bloqueio peribulbar com ropivacaína a $0,75 \%(\mathrm{G} 1)$ e ao bloqueio neuromuscular com brometo de pancurônio $(0,01 \mathrm{mg} / \mathrm{kg} \mathrm{IV})(\mathrm{G} 2)$

\begin{tabular}{lllllll}
\hline \multirow{2}{*}{ Momento } & G1 & \multicolumn{7}{c}{ G2 } & Max & Md & Min & Max \\
\cline { 2 - 7 } & Md & 2 & 3 & $2 \mathrm{aA}$ & 1 & 3 \\
\hline M2 & $3 \mathrm{aA}$ & 1 & 2 & $1 \mathrm{bA}$ & 1 & 2 \\
M3 & 1bA & 1 & 2 & $1 \mathrm{bA}$ & 1 & 2 \\
M4 & $1 \mathrm{bA}$ & 1 & 2 & $1 \mathrm{bA}$ & 1 & 2 \\
M5 & $1 \mathrm{bA}$ & 1 & 3 & $1 \mathrm{bA}$ & 1 & 2 \\
M6 & $1 \mathrm{bA}$ & 1 & 3 & $1 \mathrm{bA}$ & 1 & 3 \\
\hline
\end{tabular}

Medianas seguidas de letras distintas, minúsculas na coluna e maiúsculas na linha, diferem entre si $(\mathrm{P}<0,05)$.

M2: 20 minutos após M1 (realização do bloqueio peribulbar e administração do BNM); M3: 20 minutos após M2 (G1) e cinco minutos após M2 (G2); M4: incisão corneal; M5: remoção do cristalino; M6: início da sutura corneal; M7: término da cirurgia.

Escores: (1) olho em posição correspondente ao eixo neutro (posição desejável para cirurgia), (2) olho direcionado com leve desvio inferior (ângulo inferior a $22,5^{\circ}$ do eixo vertical), (3) correspondente a ângulo superior a $22,5^{\circ}$ (não indicado para a cirurgia). 


\section{Oliva et al.}

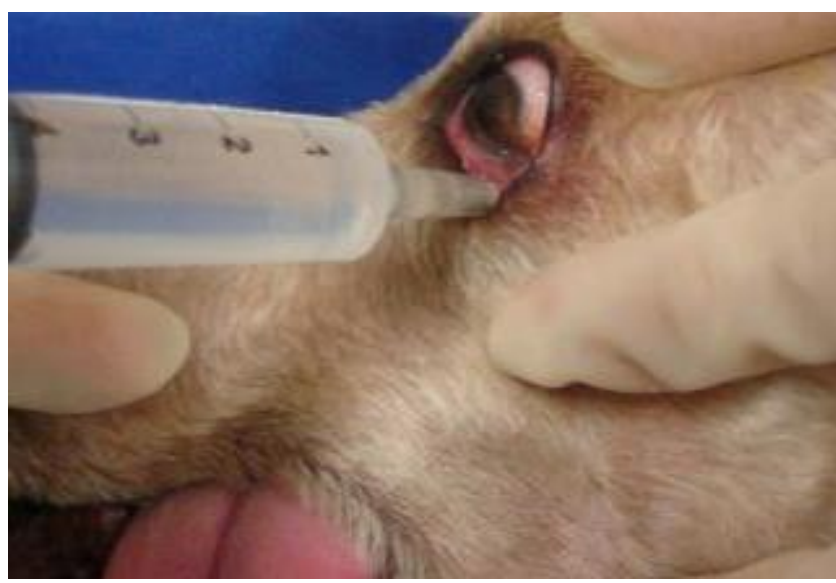

Figura 1. Bloqueio peribulbar em cão: primeiro ponto de inserção da agulha situado no terço lateral na margem orbitária inferior.

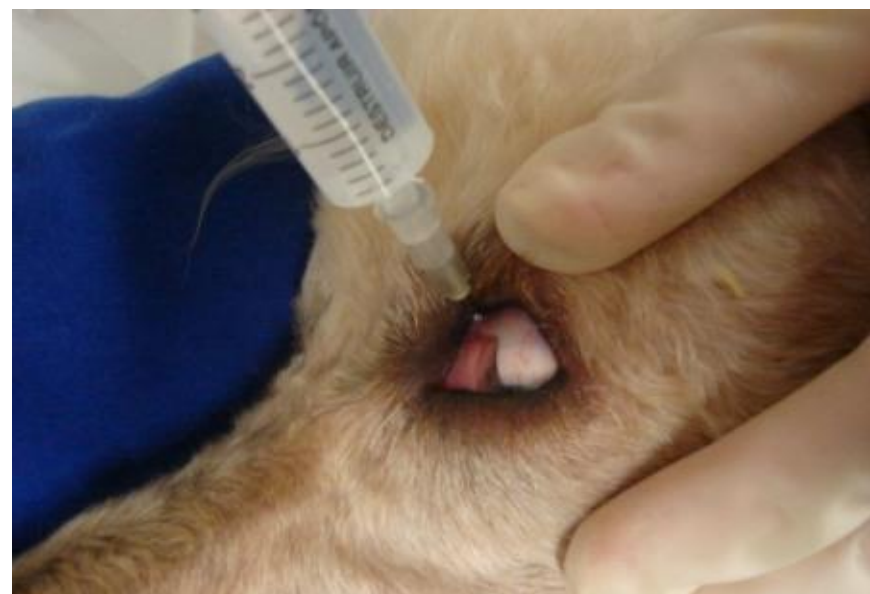

Figura 2. Bloqueio peribulbar em cão: segundo ponto de inserção da agulha situado no terço medial da margem orbitária superior. Notar marca em seringa com fio de esparadrapo para orientar a localização do bisel da agulha (direcionado para o globo ocular).

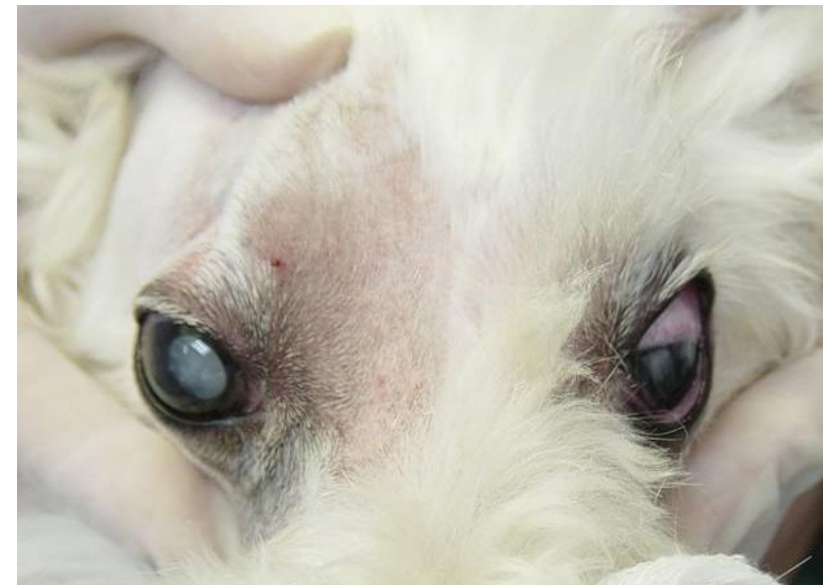

Figura 3. Centralização do globo ocular (olho direito) após a realização do bloqueio peribulbar com ropivacaína $0,75 \%$ : comparar com o olho contralateral rotacionado. 
O bloqueio neuromuscular seletivo com brometo de pancurônio também promoveu centralização do globo ocular na maioria dos pacientes, entretanto um paciente (17\%) necessitou de uma dose complementar desse fármaco para obter posicionamento ocular adequado e permitir a realização do procedimento. Esse dado difere dos resultados obtidos por Lee et al. (1998), em que todos os pacientes apresentaram escores de centralização satisfatórios durante o período experimental, com a utilização da mesma dose de brometo de pancurônio.

Os valores da PIO variaram significativamente no G1, ocorrendo redução significativa dessa variável em M3 em relação a M0. Nos pacientes do G2, observou-se estabilidade desse parâmetro em todos os momentos. Comparando-se os grupos, o bloqueio peribulbar promoveu valores de PIO significativamente menores que os promovidos pelo bloqueio neuromuscular, observado em M3 (Tab. 3). No grupo G1 foi, então, realizada a análise de regressão. Verificou-se que, a cada minuto decorrido, houve redução da PIO em 0,11010 $\mathrm{mmHg}$, de acordo com a equação de regressão linear: $\mathrm{Y}=17,00703$ - 0,011011X, sendo Y=PIO e X=momento, com coeficiente de determinação de $92,95 \%$.

Tabela 3. Média ( $\overline{\mathrm{X}})$ e erro-padrão da média (EPM) da pressão intraocular (PIO) em mmHg, em cães anestesiados pelo isofluorano em associação ao bloqueio peribulbar com ropivacaína a $0,75 \%(\mathrm{G} 1)$ e ao bloqueio neuromuscular com brometo de pancurônio $(0,01 \mathrm{mg} / \mathrm{kg}$ IV) $(\mathrm{G} 2)$

\begin{tabular}{lll}
\hline Momento & G1 $(\overline{\mathrm{X}} \pm \mathrm{EPM})$ & $\mathrm{G} 2(\overline{\mathrm{X}} \pm \mathrm{EPM})$ \\
\hline M0 & $17,33 \pm 0,38 \mathrm{~A}$ & $15,67 \pm 0,43 \mathrm{~A}$ \\
M1 & $14,50 \pm 0,74 \mathrm{~A}$ & $14,75 \pm 0,63 \mathrm{~A}$ \\
M2 & $13,96 \pm 0,53 \mathrm{~A}$ & $16,60 \pm 0,61 \mathrm{~A}$ \\
M3 & $10,67 \pm 0,59 \mathrm{~A}$ & $14,71 \pm 0,63 \mathrm{~B}$ \\
\hline
\end{tabular}

Médias seguidas de letras distintas na linha diferem entre si pelo teste Tukey $(\mathrm{P}<0,05)$.

M0: antes da medicação pré-anestésica; M1: 15 minutos após M0 (indução da anestesia, intubação traqueal e início da manutenção anestésica); M2: 20 minutos após M1 (realização do bloqueio peribulbar em G1 ou aplicação do BNM em G2); M3: 20 minutos após M2 em G1 ou cinco minutos após M2 em G2.

A redução significativa dos valores da PIO no G1 ao final da realização do bloqueio peribulbar $(-6,056 \mathrm{mmHg}$ em 55 minutos) e significativamente menor à observada no bloqueio neuromuscular no G2, pode ter sido em decorrência da capacidade da ropivacaína em influenciar o volume sanguíneo intraocular pelo seu efeito vasoconstritor próprio, como demonstrado por Ishiyama et al. (1997). Em anestesias oftálmicas no homem, esse efeito da ropivacaína sobre a PIO também foi evidenciado por Nociti et al. (2001) e Olmez et al. (2004), quando comparada a outros anestésicos locais.

A diferença significativa dos valores da PIO entre os grupos poderia, ainda, estar relacionada ao tempo adicional de anestesia geral inalatória realizada nos animais do G1, pois a espera de 20 minutos para o início da cirurgia, respeitando o período de latência da ropivacaína, prolongou o tempo anestésico. Contudo, segundo Cunningham e Barry (1986), a redução da PIO causada pelos anestésicos inalatórios halogenados é secundária à diminuição significativa nos valores de pressão arterial em planos anestésicos muito profundos, e, nos pacientes, deste estudo, a pressão arterial foi mantida nos limites de normalidade para espécie canina durante todo o período experimental.

As avaliações eletrocadiográficas não revelaram alterações no ritmo cardíaco compatíveis com a ativação do ROC nos diferentes momentos estudados em ambos os grupos. Os animais apresentaram ritmo sinusal ou arritmia sinusal, mantidos mesmo após o início da manipulação do globo ocular. No G1, este resultado foi semelhante ao observado por Déb et al. (2001), que também utilizaram a anestesia peribulbar associada à anestesia geral inalatória em crianças. No G2 este resultado foi semelhante ao obtido por Clutton et al. (1988), que demonstraram que o ROC raramente ocorre quando se utilizam técnicas de anestesia 
balanceada que incluam a utilização de agentes bloqueadores neuromusculares não despolarizantes durante a manutenção anestésica.

O bloqueio peribulbar pode apresentar uma vantagem com relação à prevenção do desencadeamento desse reflexo, pois, além de promover relaxamento da musculatura extraocular, evitando planos anestésicos profundos e manipulação excessiva do globo ocular, tem o potencial de inibir diretamente a transmissão dos estímulos aferentes para o SNC da mesma forma que o bloqueio retrobulbar.

Outros fatores importantes que contribuíram para prevenir a deflagração do ROC neste estudo foram a destreza do cirurgião, que resultou em manipulação suave do globo ocular, e a manutenção dos níveis da $\mathrm{PaCO}_{2}$ dentro dos valores da normalidade durante todo o período anestésico, os quais também são fatores aconselhados por Thurmon et al. (1996) para prevenir a ocorrência desse reflexo e, consequentemente, de equipamentos de anestesia mais sofisticados.

O bloqueio peribulbar com ropivacaína a $0,75 \%$, associado à anestesia geral inalatória com isofluorano, foi viável para a realização da facectomia extracapsular em cães, pois determinaram condições cirúrgicas desejáveis para uma cirurgia intraocular, caracterizadas por acinesia da musculatura extraocular e centralização do globo ocular satisfatórios, valores de PIO adequados e ausência de deflagração do reflexo oculocardíaco. Adicionalmente, o bloqueio sensitivo prolongado da ropivacaína promoveu analgesia pósoperatória residual.

Concluiu-se que a técnica de anestesia peribulbar no cão pode ser realizada como a descrita para o homem, reduzindo-se, contudo, de maneira significativa, os volumes anestésicos utilizados. Tal bloqueio, com a utilização de ropivacaína, é eficaz para a realização de facectomias, uma vez que compõe a anestesia balanceada com técnicas de anestesia geral, em substituição ao uso de bloqueadores neuromusculares, apresenta condições cirúrgicas superiores no que diz respeito à redução da $\mathrm{PIO}$, dispensa a ventilação controlada em condições anestésicas normais e produz analgesia residual pós-operatória.

\section{AGRADECIMENTOS}

À CAPES (Coordenação de Aperfeiçoamento de Pessoal de Nível Superior), pela concessão da bolsa de Mestrado. À Seção de Pós-graduação em Anestesiologia, da Faculdade de Medicina de Botucatu - UNESP, pelo fornecimento do material de consumo e anestésico utilizados para realização da parte experimental no presente estudo. Ao laboratório Astra Química e Farmacêutica Ltda., pelo fornecimento da ropivacaína.

\section{REFERÊNCIAS BIBLIOGRÁFICAS}

ACCOLA, P.J.; BENTLEY, E.; SMITH, L.J. et al. Development of a retrobulbar injection technique for ocular surgery and analgesia in dogs. J. Am. Vet. Med. Assoc., v.229, p.220-225, 2006.

CARREGARO, A.B.; GERARDI, P.M.; MUJALLI, M.C.L.C. et al. Uso de atracúrio para o bloqueio da musculatura extrínseca do bulbo ocular em cães submetidos à anestesia inalatória sob ventilação espontânea. Arq. Bras. Med. Vet. Zootec., v.58, p.1057-1063, 2006.

CLUTTON, R.E.; BOYD, C.; RICHARDS, D.L.S. et al. Significance of the oculocardiac reflex during ophthalmic surgery in the dog. $J$. Small Anim. Pract., v.29, p.573-579, 1988.

CUNNINGHAM, A.J.; BARRY, P. Intra-ocular pressure: physiology and implications for anesthetic management. Can. Anaesth. Soc. J., v.33, p.195-208, 1986.

DÉB, K.; SUBRAMANIAM, R.; DEHRAN, M. et al. Safety and efficay of peribulbar block as adjunct to general anaesthesia for paediatric ophthalmic surgery. Paed. Anaesth., v.11, p.161$167,2001$.

FELDMANN, H.S.; COVINO, B.G. Comparative motor-blocking effects of bupivacaine and ropivacaine, a new amino amide local anesthetic, in the rat and dog. Anesth. Analg., v.67, p.1047-1052, 1988.

GILGER, B.C. Lens. In: SLATTER, D. (Ed). Textbook of small animal surgery. Philadelphia: Saunders, 2003. p.1287-1303. 
GIOIA, L.; FANELLI, G.; CASATI, A. et al. A prospective, randomized, double-blinded comparison of ropivacaine $0,5 \%, 0,75 \%$ and $1 \%$ for peribulbar block. J. Clin. Anesth., v.16, p.184-188, 2004.

HODGSON, D.A.; DUNLOP, C.I. General anesthesia for horses with specific problems. Vet. Clin. N. Am.: Equine Pract., v.6, p.625-650, 1990.

ISHIYAMA, T.; DOHI, S.; IIDA, H. et al. The effects of topical and intravenous ropivacaine on canine pial microcirculation. Anesth. Analg., v.85, p.75-81, 1997.

KANEMAKI, N.; ONO, K.; SUGA, T. et al. Retrobulbar anesthesia of both bupivacaine and lidocaine for intra-ocular surgery in dogs. In: ANNUAL MEETING OF THE AMERICAN COLLEGE OF VETERINARY OPHTHALMOLOGISTS, 31., 2000, Montreal, Canadá. Vet. Ophthalmol., v.3, p.247257,2000 .

LEE, D.D.; MEYER, R.E.; SULLIVAN, T.C. et al. Respiratory depressant and skeletal muscle relaxant effects of low-dose pancuronium bromide in spontaneously breathing isoflurane anesthetized dogs. Vet. Anesth., v.27, p.473-479, 1998.

LING, R.; BEIGI, B.; QUINN, A. et al. Effect of Honan ballon compression on peribulbar anesthesia adequacy in cataract surgery. $J$. Cataract Refract. Surg., v.28, p.113-117, 2002.

LUDDERS, J.W. Anesthesia for the patient with central nervous system or ophthalmic disease: anesthesia for the ophthalmic surgical patient. In: SLATTER, D. (Ed). Textbook of small animal surgery. Philadelphia: WB Saunders, 2003. p. 2560-2564.

McGOLDRICK, K.E.; FEITL, M.E.; KRUPIN, $T$. Techniques of neural blockade: neural blockade for ophthalmologic surgery. In: COUSINS, M.J.; BRIDENBAUGH, P.O. (Eds). Neural blockade: in clinical anesthesia and management of pain. Philadelphia: (Lippincott Williams \& Wilkins, 2001

MORGAN, R.V. Calibration tables for the shiotz tonometer. In: Handbook of small animal practice. Philadelphia: W.B. Saunders, 1997.
NOCITI, J.R.; SERZEDO, P.S.M.; ZUCCOLOTTO, E.B. et al. Intra-ocular pressure and ropivacaine in peribulbar block: a comparative study with bupivacaine. Acta Anaesthesiolog. Scand., v.45, p.600-602, 2001.

OLMEZ, G.; CAKMAK, S.S.; CACA, I. et al. Intra-ocular pressure and quality of blockade in peribulbar anesthesia using ropivacaine or lidocaine with adrenaline: a double-blind randomized study. Tohoku J. Exp. Med., v.204, p.203-208, 2004.

PERELLO, A.; GEORGE, J.; SKELTON, V. et al. A double-blind randomised comparison of ropivacaine $0,5 \%$, bupivacaine $0,375 \%$ lidocaine $1 \%$ and ropivacine $0,5 \%$ - lidocaine $1 \%$ mixture for cataract surgery. Anaesthesiology, v.55, p.1003-1007, 2000.

SKARDA, R.T. Selected anesthetic techniques. Local and regional anesthetic and analgesic techniques: dogs. In: THURMON, J.C.; TRANQUILLI, W.J.; BENSON, G.J. (Eds). Lumb and Jones' veterinary anesthesia. Baltimore: Williams \& Wilkins, 1996. p.426447.

THURMON, J.C.; TRANQUILLI, W.J.; BENSON, G.J. (Eds). Lumb and Jones' veterinary anesthesia. Baltimore: Williams \& Wilkins, 1996. p.812-818.

TROLL, G.F. Regional ophthalmic anesthesia: safe techniques and avoidance of complications. J. Clin. Anesth., v.7, p.163-172, 1995.

VANETTI, L.F.A. Anestesia para oftalmologia. In: YAMASHITA, A.M.; TAKAOKA, F.; AULER JUNIOR, J.O.C. (Eds). Anestesiologia SAESP. São Paulo: Atheneu, 2001. p.811-830.

VÁSQUEZ, C.E.; MACUCO, M.V.; BEDIN, A. et al. Comparação da qualidade do bloqueio oftálmico periconal com ropivacaína a $1 \%$ e $0,75 \%$ com punção dos pontos infraorbitário lateral e medial da órbita. Rev. Bras. Anestesiol., v.52, p.681-688, 2002. 